REPRESENTATION THEORY

An Electronic Journal of the American Mathematical Society

Volume 14, Pages 627-644 (October 1, 2010)

S $1088-4165(2010) 00391-X$

\title{
AUTOMATA AND CELLS IN AFFINE WEYL GROUPS
}

\author{
PAUL E. GUNNELLS
}

\begin{abstract}
Let $\widetilde{W}$ be an affine Weyl group, and let $C$ be a left, right, or two-sided Kazhdan-Lusztig cell in $\widetilde{W}$. Let $\operatorname{Red}(C)$ be the set of all reduced expressions of elements of $C$, regarded as a formal language in the sense of the theory of computation. We show that $\operatorname{Red}(C)$ is a regular language. Hence, the reduced expressions of the elements in any Kazhdan-Lusztig cell can be enumerated by a finite state automaton.
\end{abstract}

\section{INTRODUCTION}

1.1. Let $W$ be a Coxeter group with generating set $S$. In their work on Coxeter groups and Hecke algebras, Kazhdan and Lusztig defined a partition of $W$ into sets called cells. When $W$ is a Weyl or affine Weyl group, it is known that cells have deep connections with many areas of algebra and geometry, such as singularities of Schubert varieties [26, representations of $p$-adic groups [28, characters of finite groups of Lie type 29, and the geometry of unipotent conjugacy classes in simple complex algebraic groups [7, 8].

The definition of cells is quite complicated (\$2.2). It involves the construction of a subtle equivalence relation on $W$ built from both easy and difficult combinatorial data. In particular, from the definition it is not clear how "computable" cells are. For instance, it is highly nontrivial to decide whether two elements of $W$ lie in the same cell or not, or to characterize all elements in a given cell. Nevertheless, in all known examples where cells have been explicitly computed, one sees that cells ultimately have a relatively simple geometric and combinatorial structure. We refer to [12, 21] for examples and further discussion of this phenomenon.

1.2. This paper addresses the following computational problem: Given a cell $C$ in a Coxeter group $W$, how can we encode the (typically) infinite amount of data represented by $C$ with a finite structure? In this generality, this question was first considered by Casselman, who phrased an answer in terms of finite state automata (\$2.4). More precisely, let $\operatorname{Red}(W)$ be the set of all reduced expressions of all elements of $W$, considered as a subset of the free monoid on the generating set $S$. We regard $\operatorname{Red}(W)$ as a formal language in the sense of the theory of computation. Given a cell $C$, let $\operatorname{Red}(C) \subset \operatorname{Red}(W)$ be the set of all reduced expressions of all $w \in C$. Then we have the following conjecture of Casselman:

Received by the editors September 5, 2008 and, in revised form, July 27, 2009.

2010 Mathematics Subject Classification. Primary 20F10, 20 F55.

Key words and phrases. Kazhdan-Lusztig cells, finite state automata, regular languages, affine Weyl groups, Coxeter hyperplane arrangements. 
1.3. Conjecture. For any Coxeter group $W$ and any cell $C \subset W$, there exists a finite state automaton accepting the language $\operatorname{Red}(C)$. That is, the language $\operatorname{Red}(C)$ is regular.

The precise definitions of the terms in Conjecture 1.3 are given in $\$ 2.4$, here we give an informal sense of what the conjecture means.

A finite state automaton is a simple theoretical model of a computer. It has finite memory and can complete only one task: acceptance/rejection of its input. More precisely, given a word $a_{1} \cdots a_{k}$ on some alphabet, an automaton reads the word from left to right, and while doing so moves through finitely many memory states. After reading the word, the automaton decides based on which state it occupies whether or not to accept the word or throw it away. A language is called regular if one can find a finite state automaton accepting exactly the words in the language.

Thus Casselman's conjecture implies that given any cell $C$, there exists a simple machine $\mathscr{A}(C)$ that decides whether or not $w \in W$ lies in $C$ simply by reading through a reduced expression $s_{1} \cdots s_{k}$ for $w$. The finiteness of the automaton implies that the decision is only based on finitely many patterns appearing in the expression. One can also use $\mathscr{A}(C)$ to systematically list all reduced expressions of all elements of $C$. We remark that the first connection between automata and Kazhdan-Lusztig cells appears (implicitly) in the work of Bédard 2, 3, 4. In fact, Bédard used an infinite regular language to prove for the first time that certain Coxeter groups have an infinite number of left cells.

Work of Brink and Howlett 11] shows that the language $\operatorname{Red}(W)$ of all reduced expressions of all elements of $W$ is regular, although this does not prove Conjecture 1.3. a sublanguage of a regular language need not be regular. Indeed, it is not even clear how one can use the tools underlying the fundamental results of Brink and Howlett to attack Conjecture 1.3. Moreover, Conjecture 1.3 does not specify what structures in $W$ should be used to build the machines $\mathscr{A}(C)$.

Casselman's conjecture is trivially true for any finite Coxeter group, in particular for Weyl groups, since for such groups the language $\operatorname{Red}(W)$ is obviously finite. The first interesting infinite example of Conjecture 1.3 follows from work of Shi 34] on Kazhdan-Lusztig cells and Eriksson [18] and Headley [22] on automata. More precisely, let $W=\widetilde{A}_{n}$, the affine Weyl group of type $A$. Shi showed that $W$ can be partitioned into finitely many geometrically defined subsets, called sign-type regions, such that each left cell $C$ of $W$ is a union of finitely many such regions. Headley showed that the sign-type regions can be used as a set of states for an automaton $\mathscr{A}$ recognizing $\operatorname{Red}(W)$. Together these results imply Conjecture 1.3 for $\widetilde{A}_{n}$, since given $C$ one can modify $\mathscr{A}$ to only accept the reduced expressions corresponding to elements of $C$.

1.4. In this paper we prove Conjecture 1.3 when the Coxeter group is an affine Weyl group $\widetilde{W}$ (Theorem 4.6). The proof uses two ingredients.

The first is a family of finite state automata $\mathscr{A}_{N}, N \in \mathbb{Z}_{\geq 0}$, each of which recog$\operatorname{nizes} \operatorname{Red}(\widetilde{W})$. The construction generalizes work of Eriksson [18] and Headley [22]. Each $\mathscr{A}_{N}$ is built from the complement of a certain affine hyperplane arrangement $\mathscr{H}_{N}$.

The second is a result of Du [16], who proved that each left cell $C$ of $\widetilde{W}$ can be represented as the union of a finite set of convex polyhedra of a certain type. We show that if $N \gg 0$, then we can write each of these polyhedra as a finite union of 
regions in the complement of $\mathscr{H}_{N}$. This allows us to define $\mathscr{A}(C)$ by identifying the set of states of $\mathscr{A}_{N}$ that correspond exactly to the reduced expressions of elements of $C$.

\section{BACKGROUND}

2.1. In this section we recall background and standard notation. For more details and proofs we refer to 9, 24 for Coxeter groups, 9, 25] for Kazhdan-Lusztig cells, and 23] for automata and formal languages.

Let $\Phi$ be an irreducible, reduced root system, and let $W$ be the associated Weyl group. Decompose $\Phi$ into a union of positive and negative roots $\Phi^{+} \cup \Phi^{-}$. Let $\Delta \subset \Phi^{+}$be the simple roots and let $S \subset W$ be the corresponding subset of generators in the presentation of $W$ as a Coxeter group. For each $s \in S$, we write $\alpha_{s} \in \Delta$ for the associated simple root. Conversely, given a simple root $\alpha$, we write $s_{\alpha} \in S$ for the associated generator.

We assume $\Phi$ spans a real vector space $V$ equipped with a $W$-invariant inner product ( , ). The group $W$ acts on $V$ as usual: if $\alpha \in \Phi$, then we have the reflection

$$
v \longmapsto v-\frac{2(v, \alpha)}{(\alpha, \alpha)} \alpha,
$$

which maps $W$ faithfully onto a subgroup of GL $(V)$. We will write this reflection action as a right action: $v \mapsto v \cdot s_{\alpha}$. Let $\mathscr{C}^{+}$be the positive Weyl chamber determined by $\Delta$.

The root system $\Phi$ has a unique highest root $\widetilde{\alpha}$. By definition $\widetilde{\alpha}$ has the property that for any $\beta \in \Phi$, the difference $\widetilde{\alpha}-\beta$ can be written as a nonnegative linear combination of the simple roots. One also knows [10, VI, §1.8, Prop. 25(iv)] that for any positive root $\beta \neq \widetilde{\alpha}$, we have

$$
\frac{2(\beta, \widetilde{\alpha})}{(\widetilde{\alpha}, \widetilde{\alpha})} \in\{0,1\}
$$

Let $\alpha \in \Phi$ and $k \in \mathbb{Z}$. Let $H_{\alpha, k} \subset V$ be the affine hyperplane

$$
H_{\alpha, k}=\{v \in V \mid(\alpha, v)=k\},
$$

and let $H_{\alpha, k}^{1}$ be the subset

$$
H_{\alpha, k}^{1}=\{v \in V \mid k \leq(\alpha, v) \leq k+1\} .
$$

Attached to the hyperplane $H_{\alpha, k}$ is the affine reflection $s_{\alpha, k}$, which acts on $V$ by

$$
s_{\alpha, k}: v \longmapsto v-2((\alpha, v)-k) \alpha /(\alpha, \alpha) .
$$

The affine Weyl group $\widetilde{W}$ corresponding to $\Phi$ is the group generated by all the affine reflections $s_{\alpha, k}$. We can represent $\widetilde{W}$ as a finitely generated Coxeter group by using the generating set $\widetilde{S}=S \cup\left\{s_{\widetilde{\alpha}, 1}\right\}$, where we identify $s_{\alpha} \in S$ with $s_{\alpha, 0}$.

Let $\mathscr{H}$ be the affine hyperplane arrangement consisting of all affine hyperplanes of the form (11). The connected components of $V \backslash \mathscr{H}$ are called alcoves. There is a distinguished alcove $A_{0}$ defined by

$$
A_{0}=\left\{v \mid 0<(\alpha, v)<1 \quad \text { for all } \alpha \in \Phi^{+}\right\},
$$

and $w \mapsto A_{0} \cdot w$ gives a bijection between $\widetilde{W}$ and the set of alcoves. We often identify alcoves and elements of $\widetilde{W}$ under this bijection. 
Any $w \in \widetilde{W}$ determines a function $b_{w}: \Phi^{+} \rightarrow \mathbb{Z}$ as follows. The closure of the alcove $A_{0} \cdot w$ can be uniquely written as the intersection of subsets of the form (2):

$$
\overline{A_{0} \cdot w}=\bigcap_{\alpha \in \Phi^{+}} H_{\alpha, k(\alpha)}^{1} .
$$

We put $b_{w}(\alpha)=k(\alpha) \in \mathbb{Z}$.

An expression for $w \in \widetilde{W}$ is a representation of $w$ as a product of elements of $\widetilde{S}$. An expression is reduced if it has minimal length among all expressions for $w$. We define the length $\ell(w)$ of $w$ to be the length of a reduced expression for $w$.

Given an expression $s_{1} \cdots s_{N}$, a subexpression is a (possibly empty) expression of the form $s_{i_{1}} \cdots s_{i_{M}}$, where $1 \leq i_{1}<\cdots<i_{M} \leq N$. We endow $\widetilde{W}$ with a partial order by defining $u \leq w$ if an expression for $u$ appears as a subexpression of a reduced expression for $w$.

2.2. Next we describe Kazhdan-Lusztig cells. Given $w \in \widetilde{W}$, we define the left descent set $L(w) \subset \widetilde{S}$ by

$$
L(w)=\{s \in \widetilde{S} \mid \ell(s w)<\ell(w)\},
$$

and analogously define the right descent set $R(w)$ by the condition $\ell(w s)<\ell(w)$.

Let $u, w \in \widetilde{W}$, and let $P_{u, w}(t) \in \mathbb{Z}[t]$ be the Kazhdan-Lusztig polynomial attached to the pair $(u, w)$ 25. We do not recall the definition here, but only mention the following properties:

(1) $P_{u, w}=0$ unless $u \leq w$,

(2) $P_{u, u}=1$, and

(3) $P_{u, w}$ has degree at most $d(u, w):=(\ell(w)-\ell(u)-1) / 2$.

We write $u-w$ if $u<w$ and $\operatorname{deg} P_{u, w}=d(u, w)$. If $w<u$ we write $u-w$ if $w-u$ holds.

We are now ready to define the cells of $\widetilde{W}$. The left $W$-graph $\Gamma_{L}$ of $\widetilde{W}$ is the directed graph with vertex set $\widetilde{W}$, and with an arrow from $u$ to $w$ if and only if $u-w$ and $L(u) \not \subset L(w)$. We can similarly define the right $W$-graph $\Gamma_{R}$. Then the left and right cells of $\widetilde{W}$ are extracted from the graphs $\Gamma_{L}, \Gamma_{R}$ as follows. Recall that given any directed graph, we say two vertices are in the same strong connected component if there exist directed paths from each vertex to the other.

2.3. Definition. The left cells (respectively, right cells) of $\widetilde{W}$ are the strong connected components of the graph $\Gamma_{L}$ (resp., $\Gamma_{R}$ ). The elements $u, w \in \widetilde{W}$ are in the same two-sided cell if they are in the same left or right cell.

It is known that each affine Weyl group has only finitely many two-sided cells, and that each two-sided cell is a union of finitely many left cells [31]. We remark that in general the graphs $\Gamma_{L}, \Gamma_{R}$ are extremely complicated, although they do show many regular features (and in some cases are perhaps amenable to analysis using finite state automata). Figures 810 at the end of this paper show part of the subgraph of $\Gamma_{L}$ corresponding to a particular left cell of $\widetilde{G}_{2}$. The labels of the figure show the length difference $\ell=\ell(w)-\ell(u)$ of the words connected by edges in the graph; we have omitted the arrowheads for clarity. 
2.4. Finally, we discuss automata. Let $A$ be a finite set, and let $A^{*}$ be the free monoid generated by $A$ under the operation of concatenation. Thus $A^{*}$ consists of all sequences $a_{1} \cdots a_{k}$, where $a_{i} \in A$, together with the empty sequence. A formal language $L$ is any subset of $A^{*}$. We call $A$ the alphabet for the language $L$. Among all formal languages, our interest lies in the regular languages, which are defined using finite state automata:

2.5. Definition. A finite state automaton $\mathscr{A}$ over the alphabet $A$ consists of the following data:

(1) a finite set $Q$, called the set of states,

(2) a unique initial state $q_{0} \in Q$,

(3) a subset $F \subset Q$, called the set of accepting states, and

(4) a function $t: Q \times A \rightarrow Q \cup\{\varnothing\}$, called the transition function.

A word $a_{1} \cdots a_{k} \in A^{*}$ is said to be accepted by $\mathscr{A}$ if there exist states $q_{0}, \ldots, q_{k}$ such that $t\left(q_{i}, a_{i+1}\right)=q_{i+1}$ for $0 \leq i \leq k-1$, and $q_{k} \in F$. A language $L$ is regular if there exists a finite state automaton accepting exactly the words in $L$.

We may picture $\mathscr{A}$ as a decorated directed graph as follows. We label the vertices of the graph by $Q$, and circle the vertices corresponding to the final states. We put an edge from $q$ to $q^{\prime}$ labelled by $a \in A$ if $t(q, a)=q^{\prime}$. A path in this graph starting at $q_{0}$ determines a word in $A^{*}$ by concatenating the edge labels as one traverses the path. A word is accepted by $\mathscr{A}$ if and only if one can find a path building the word that ends in an accepting state.

\section{The automaton}

3.1. Let $\operatorname{Red}(\widetilde{W})$ be the set of all reduced expressions for all elements $w \in \widetilde{W}$, regarded as a language on the alphabet $\widetilde{S}$. More generally, if $U$ is any subset of $\widetilde{W}$, we let $\operatorname{Red}(U)$ be the set of all reduced expressions for all $w \in U$.

By work of Brink and Howlett [11, it is known that $\operatorname{Red}(\widetilde{W})$ is regular 1 Our goal is to show the regularity of the language $\operatorname{Red}(C)$ for any cell of $\widetilde{W}$. To do this we generalize an automaton for affine Weyl groups first described by Eriksson [18] and Headley 22.

3.2. Let $\mathscr{H} \subset V$ be a finite affine hyperplane arrangement, and let $\mathscr{R}$ be the set of connected components of $V \backslash \mathscr{H}$. We will always assume that $\mathscr{H}$ contains the hyperplanes $H_{\widetilde{\alpha}, 1}$ and $H_{\alpha, 0}, \alpha \in \Delta$, which we denote by $\left\{H_{s} \mid s \in \widetilde{S}\right\}$.

3.3. Definition. We say the set of regions $\mathscr{R}$ has property $(*)$ if it satisfies the following condition:

If $R \in \mathscr{R}$ and $A_{0}$ lie on the same side of $H_{s}$, then there is a unique $R^{\prime} \in \mathscr{R}$ such that $R \cdot s \subset R^{\prime}$.

The following result explains the connection between property $(*)$ and automata:

3.4. Proposition. Suppose the set of connected components $\mathscr{R}$ of a finite affine hyperplane arrangement $\mathscr{H}$ satisfies $(*)$. Then there is a finite state automaton $\mathscr{A}$ accepting $\operatorname{Red}(\widetilde{W})$ with states given by $\mathscr{R}$.

\footnotetext{
${ }^{1}$ In fact, [1] shows that the language of reduced expressions is regular for any Coxeter group.
} 
Proof. We define $\mathscr{A}$ by taking its states to be $\mathscr{R}$ and its initial state to be $A_{0}$; note that $A_{0} \in \mathscr{R}$ since $\mathscr{H}$ contains $\left\{H_{s} \mid s \in \widetilde{S}\right\}$. We declare all states to be accepting. We define the transition function $t$ by $t(R, s)=\varnothing$ unless $A_{0}$ and $R$ lie on the same side of $H_{s}$, in which case we put $t(R, s)=R^{\prime}$, where $R^{\prime}$ is the unique region with $R \cdot s \subset R^{\prime}$.

The proof that $\mathscr{A}$ accepts $\operatorname{Red}(\widetilde{W})$ is essentially the same as that for Theorem V.6 in 22. For the convenience of the reader, we give the details. First, it is clear that the unique expression for the identity in $\widetilde{W}$ is accepted. Also, all expressions of length 1 are accepted, and such expressions are automatically reduced.

Now suppose that the reduced expression $w=s_{k} \cdots s_{1}$ is accepted and let $s \in \widetilde{S}$. We must show that $s_{k} \cdots s_{1} s$ is accepted if and only if it is reduced. Recall that $s_{k} \cdots s_{1}$ is reduced if and only if the hyperplanes

$$
H_{1}, H_{2} \cdot s_{1}, H_{3} \cdot s_{2} s_{1}, \ldots, H_{k} \cdot s_{k-1} \cdots s_{1}
$$

are distinct and separate $A_{0}$ from $A_{0} \cdot w$ (cf. 24, $\$ 4.5$ ]; here we abbreviate $H_{s_{i}}$ by $\left.H_{i}\right)$. Thus if $w s$ is reduced, then $H_{s}$ must separate $A_{0}$ from $A_{0} \cdot w s$. This means $H_{s}$ does not separate $A_{0}$ from $A_{0} \cdot w$, which implies $s_{k} \cdots s_{1} s$ is accepted by $\mathscr{A}$.

Conversely, suppose $s_{k} \cdots s_{1} s$ is accepted by $\mathscr{A}$. Then $H_{s}$ does not separate $A_{0}$ from $A_{0} \cdot w$, which means $H_{s}$ separates $A_{0}$ from $A_{0} \cdot w s$. Certainly the hyperplanes obtained by applying $s$ (on the right) to (3) are distinct, and none equal $H_{s}$. Moreover, if some hyperplane $H=H_{j} \cdot s_{j-1} \cdots s_{1} s$ from this new list fails to separate $A_{0}$ from $A_{0} \cdot w s$, then $H \cdot s$ fails to separate $A_{0}$ from $A_{0} \cdot w s$, and thus cannot be part of (3). This completes the proof.

The automaton from Proposition 3.4 also satisfies the following property: if an expression $w=s_{1} \cdots s_{k}$ is reduced and is accepted by the state $R$, then the alcove $A_{0} \cdot w$ lies in the region $R$. Later, in Example 5.3, we exhibit an automaton built from a collection of regions satisfying $(*)$.

3.5. Now let $N \geq 0$ be an integer, and let $\mathscr{H}_{N}$ be the arrangement

$$
\mathscr{H}_{N}=\left\{H_{\alpha, k} \mid \alpha \in \Phi^{+}, k=-N, \ldots, N+1\right\} .
$$

Let $\mathscr{R}_{N}=V \backslash \mathscr{H}_{N}$. Then we have the following theorem:

3.6. Theorem. The set of regions $\mathscr{R}_{N}$ has the property $(*)$, and thus the automaton built from $\mathscr{R}_{N}$ using Proposition 3.4 accepts the language $\operatorname{Red}(\widetilde{W})$.

Proof. The proof is a generalization of [22, Lemma V.5]. For each $\alpha \in \Phi^{+}$, and for $k=-N-1, \ldots, N+1$ define "root strips" by

$$
R_{\alpha}^{k}= \begin{cases}\{v \mid N+1<(\alpha, v)\} & k=N+1, \\ \{v \mid(\alpha, v)<-N\} & k=-N-1, \\ \{v \mid k<(\alpha, v)<k+1\} & \text { otherwise. }\end{cases}
$$

The elements of $\mathscr{R}_{N}$ are just the connected components of all possible intersections of the $R_{\alpha}^{k}$.

Let $R \in \mathscr{R}_{N}$, and suppose $R$ and $A_{0}$ lie on the same side of $H_{s}$. We must show that $R \cdot s$ is contained in a unique element of $\mathscr{R}_{N}$. By the above discussion, we know that for each $\alpha \in \Phi^{+}$, we have $R \subset R_{\alpha}^{k}$ for some $k$ depending on $\alpha$. We must show that $R \cdot s \subset R_{\beta}^{l}$ for some $\beta \in \Phi^{+}$and some $l$.

First assume $s$ is not the affine reflection $s_{\widetilde{\alpha}, 1}$. Then either $\alpha=\alpha_{s}$ or $\alpha \neq \alpha_{s}$. In the first case, if $R \subset R_{\alpha}^{k}$, then we must have $k \geq 0$, and it is clear that $R \cdot s \subset R_{\alpha}^{-k-1}$. 


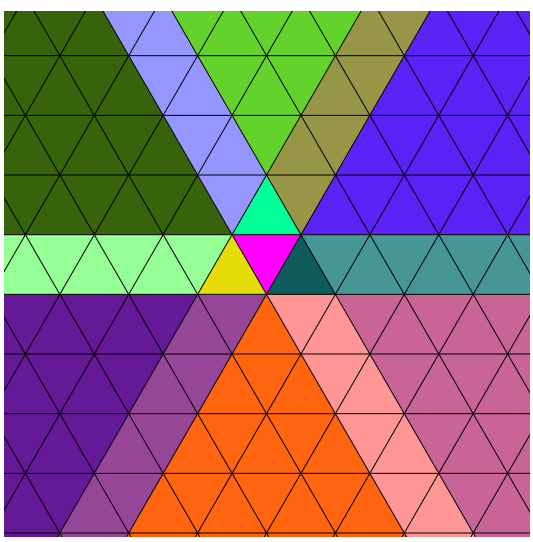

FiguRE 1. The regions in $\mathscr{R}_{0}$ for $\widetilde{A}_{2}$

Note that the hypothesis that $R$ and $A_{0}$ lie on the same side of $H_{s}$ is essential, since $R_{\alpha}^{-N-1} \cdot s$ meets both $R_{\alpha}^{N}$ and $R_{\alpha}^{N+1}$.

Next, suppose $\alpha \neq \alpha_{s}$. Then if $k \neq N+1,-N-1$, we have

$$
\begin{aligned}
R_{\alpha}^{k} \cdot s & =\{v \mid k<(\alpha \cdot s, v)<k+1\} \\
& =R_{\alpha \cdot s}^{k} .
\end{aligned}
$$

Moreover, $\alpha \cdot s \in \Phi^{+} \backslash\left\{\alpha_{s}\right\}$, since the only positive root $s$ makes negative is $\alpha_{s}$. Hence, in this case $R \cdot s$ is taken into a unique region of $\mathscr{R}_{N}$ if $s$ is not the affine reflection; if $k=N+1$ or $-N-1$, we argue similarly.

Finally, assume $s$ is the affine reflection, and suppose $R \subset R_{\alpha}^{k}$. Again, there are two possibilities to consider. If $\alpha$ is the highest root $\widetilde{\alpha}$, then since $R$ lies on the same side of $H_{s}$ as $A_{0}$, we must have $k \leq 0$. Therefore, $R \cdot s \subset R_{\alpha}^{-k+1}$. On the other hand, if $\alpha$ is not the highest root, then $c=2(\alpha, \widetilde{\alpha}) /(\widetilde{\alpha}, \widetilde{\alpha})$ either equals 0 or 1. If $c=0$, then $s_{\widetilde{\alpha}, 1}$ stabilizes all the sets $R_{\alpha}^{k}$. If $c=1$, then $s_{\widetilde{\alpha}, 1}(\alpha)=\alpha-\widetilde{\alpha}$, which is negative. We have for $k \neq N+1$,

$$
\begin{aligned}
R_{\alpha}^{k} \cdot s & =\{v \mid k<(\alpha-\widetilde{\alpha}, v)<k+1\} \\
& =\left\{v \mid-k-1<\left(\alpha^{\prime}, v\right)<-k\right\} \\
& =R_{\alpha^{\prime}}^{-k-1},
\end{aligned}
$$

where $\alpha^{\prime} \in \Phi^{+}$is the root $\widetilde{\alpha}-\alpha$. If $k=N+1$, then $R_{\alpha}^{N+1} \cdot s \subset R_{\alpha^{\prime}}^{-N-1}$. This completes the proof.

3.7. Remark. Recall that $\Phi$ is simply-laced if all roots have the same length. Suppose $\Phi$ is not simply-laced, and partition the positive roots $\Phi^{+}$into the long and short roots $\Phi_{l}^{+} \cup \Phi_{s}^{+}$. Choose a function $\nu: \Phi^{+} \rightarrow \mathbb{Z}_{\geq 0}$ that is constant on $\Phi_{l}^{+}, \Phi_{s}^{+}$, and consider the affine arrangement $\mathscr{H}_{\nu}$ consisting of the hyperplanes

$$
\mathscr{H}_{\nu}=\left\{H_{\alpha, k} \mid k=-\nu(\alpha), \ldots, \nu(\alpha)+1\right\} .
$$

Then the proof of Theorem 3.6 actually shows that the set of regions of the complement $V \backslash \mathscr{H}_{\nu}$ has property $(*)$ as well, and thus can be used as the set of states for an automaton recognizing $\operatorname{Red}(\widetilde{W})$. Indeed, the only observations one needs to 
make are that the action of $W$ preserves root lengths, and that if $2(\alpha, \widetilde{\alpha}) /(\widetilde{\alpha}, \widetilde{\alpha})=1$, then $\alpha-\widetilde{\alpha}$ has the same length as $\alpha$.

3.8. Remark. The arrangement $\mathscr{H}_{N}$ is known in the combinatorics literature as the extended Shi arrangement 33. The case $N=0$ was studied by Shi 35, who called elements of $\mathscr{R}_{0}$ sign-type regions. Figure 1 shows these regions and the alcoves for $\widetilde{A}_{2}$. Shi showed that the number of regions in $\mathscr{R}_{0}$ equals $(h+1)^{r}$, where $h$ is the Coxeter number of $\Phi$ and $r$ is the rank of $\Phi$, and also used sign-type regions to explicitly describe the Kazhdan-Lusztig cells for $\widetilde{A}_{n}$ 34. For the extended Shi arrangement, Athanasiadis [1] showed that the number of regions in $\mathscr{R}_{N-1}$ is $(N h+1)^{r}$ if $\Phi$ is of classical type, i.e., type $A, B, C, D$.

\section{Kazhdan-Lusztig CELls AND AUtomata}

4.1. In this section we prove Theorem 4.6. The main idea of the proof is to show that we can choose $N$ sufficiently large such that for any left cell $C$, we can find a finite set of regions $\left\{R_{i}\right\} \subset \mathscr{R}_{N}$ such that the alcoves in the union of the $R_{i}$ are exactly those in $C$. The automaton accepting $\operatorname{Red}(C)$ is then given by modifying the automaton from Theorem 3.6 to make only the states corresponding to the $R_{i}$ accepting.

Figure 2 shows an example for $\widetilde{W}=\widetilde{G}_{2}$. The dark lines are the hyperplanes in $\mathscr{H}_{1}$; each region of a given color is a two-sided cell, and the connected regions of a given color are the left cells. Note that we have not drawn the alcoves. It is clear from the figure that any left cell is a union of regions from $\mathscr{R}_{1}$. Note that $N=1$ is the smallest value we can take for this to work; in particular, left cells in $\widetilde{G}_{2}$ are not unions of sign-type regions.

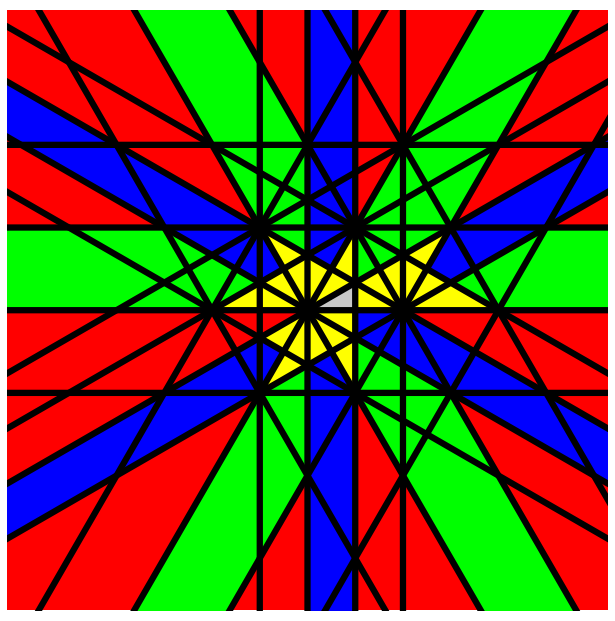

FiguRE 2. The left cells of $\widetilde{G}_{2}$ and the regions in $\mathscr{R}_{1}$ 
4.2. The main tool we need to carry out the proof is a result of $\mathrm{Du}$ [16, who showed that left cells are finite unions of certain polyhedra in $V$. More precisely, let $\mathscr{P}$ be the set of all polyhedra in $V$ of full dimension bounded by finitely many affine hyperplanes of the form $H_{\alpha, k}, \alpha \in \Phi^{+}, k \in \mathbb{Z}$. Then we have the following theorem:

4.3. Theorem ([16). Let $C$ be a left cell of $\widetilde{W}$. Identify $C$ with the closure of the set of alcoves $\left\{A_{0} \cdot w \mid w \in C\right\}$ in $V$. Then there exists a finite subset $\mathscr{P}(C) \subset \mathscr{P}$ such that

$$
C=\bigcup_{P \in \mathscr{P}(C)} P
$$

We give some indications of the proof of Theorem 4.3. Let $P \in \mathscr{P}$. Assume there exists a Weyl chamber $\mathscr{C}$ containing $P$. Let $\Delta^{\prime} \subset \Phi$ be the simple system determined by $\mathscr{C}$ (so that all $\alpha \in \Delta^{\prime}$ are nonnegative on $\mathscr{C}$ ). Then the dimension $\operatorname{dim} P$ of $P$ is defined to be the cardinality of the set?

$$
\left\{\alpha \in \Delta^{\prime} \mid \alpha \text { is unbounded on } P\right\} \text {. }
$$

Du defines a certain class of polyhedra in $\mathscr{P}$ called special polyhedra as follows. Recall (2) that we have defined the subsets $H_{\alpha, k}^{1}$. Fixing $\mathscr{C}$, we let $H_{\alpha, k}^{+}$be the closure of the unique component of $V \backslash H_{\alpha, k}$ that meets any translate of $\mathscr{C}$. Given $\Delta^{\prime}$ as above we choose a function $b: \Delta^{\prime} \rightarrow \mathbb{Z}_{\geq 0}$ and a subset $\Lambda \subset \Delta^{\prime}$. Let $\bar{\Lambda}=\Delta^{\prime} \backslash \Lambda$. Then the special polyhedron $P(\Lambda, b)$ is defined by

$$
P(\Lambda, b)=\left(\bigcap_{\alpha \in \Lambda} H_{\alpha, b(\alpha)}^{1}\right) \cap\left(\bigcap_{\alpha \in \bar{\Lambda}} H_{\alpha, b(\alpha)}^{+}\right) .
$$

Clearly, any special polyhedron lies in $\mathscr{P}$, and so does any polyhedron built by forming intersections of special polyhedra with subsets of the form $H_{\alpha, k}^{1}$ and $H_{\alpha, k}^{+}$. Du proves the following properties of special polyhedra:

(1) For any special polyhedron $P$, we can find a subpolyhedron $P^{\prime} \subset P$, also special, with $P \backslash P^{\prime}$ a finite union of special polyhedra $P_{i}$ of lower dimension than that of $P$.

(2) The polyhedron $P^{\prime}$ can be chosen such that $P^{\prime}$ is a finite union of polyhedra $Q_{j} \in \mathscr{P}$ (not special in general), and such that each $Q_{j}$ is contained in a single left cell. These $Q_{j}$ are built using intersections of $P$ with subsets of the form $H_{\alpha, k}^{1}$ and $H_{\alpha, k}^{+}$.

Finally, Du completes the proof by induction and by choosing a finite collection of special polyhedra such that all alcoves will eventually be accounted for by the $Q_{j}$ constructed in property (2). For this he uses the sign-type regions $\mathscr{R}_{0}$. Given any sign-type region $X$, he shows that there is a canonical special polyhedron $P(X) \supset X$ attached to $X$ with $\operatorname{dim} X=\operatorname{dim} P(X)$, and such that $P(X) \backslash X$ is a finite union of sign-type regions. The sign-type regions account for all alcoves, and thus so do the special polyhedra $P(X)$. Using induction on $\operatorname{dim} X$ and properties (1) and (2) completes the proof.

\footnotetext{
${ }^{2}$ We remark that there is a typo in [16], in which "unbounded" is replaced by "bounded" in the definition.
} 
The most subtle part of the proof of Theorem 4.3 is, given a special polyhedron $P$, the construction of $P^{\prime}$ and the polyhedra $Q_{j}$. For this Du constructs certain infinite sequences $y_{1}, y_{2}, \ldots, y_{i}, \ldots$ in special polyhedra that become left-equivalent for $i$ sufficiently large. A key ingredient here is the boundedness of Lusztig's $a$ function for affine Weyl groups [30].

Returning to the general discussion, here is the connection between polyhedra from $\mathscr{P}$ and the regions $\mathscr{R}_{N}$ :

4.4. Lemma. Let $\left\{P_{i}\right\}$ be a finite subset of $\mathscr{P}$, and let $P$ be the (not necessarily convex) union of the $P_{i}$. Then for $N$ sufficiently large, we can find a finite set of regions $\mathscr{R}(P) \subset \mathscr{R}_{N}$ such that

$$
P=\bigcup_{R \in \mathscr{R}(P)} \bar{R}
$$

Proof. First, we assume that $P$ is a single polyhedron from $\mathscr{P}$. If $P$ is bounded, then $P$ is the closure of a finite union of alcoves. Since any finite collection of alcoves can appear as regions in $\mathscr{R}_{N}$ for $N \gg 0$, the statement follows.

If $P$ is unbounded, then there are only finitely many positive roots $\alpha$ such that $(\alpha, v) \geq k(\alpha)$ is a defining inequality for $P$, where $k(\alpha) \in \mathbb{Z}$. Then we simply choose

$$
N \gg \max _{\alpha \in \Phi^{+}}|k(\alpha)|,
$$

and $P$ can be written as a union of regions from $\mathscr{R}_{N}$.

Finally, if $P=\bigcup P_{i}$ is a union of polyhedra, then we compute $N_{i}$ for each $P_{i}$ as above and then choose $N \gg \max N_{i}$. This completes the proof.

4.5. We are now ready to prove our main theorem:

4.6. Theorem. Let $C$ be a left cell in $\widetilde{W}$, and let $\operatorname{Red}(C)$ be the language of all reduced expressions of $w \in C$. Then $\operatorname{Red}(C)$ is regular.

Proof. By Theorem 4.3 the cell $C$ is a union $P=\bigcup P_{i}$ of finitely many polyhedra from $\mathscr{P}$. By Lemma 4.4, we may find $N \gg 0$ such that each $P_{i}$ is a union of the closures of regions of $\mathscr{R}_{N}$. Let $\mathscr{A}_{N}$ be the automaton from Theorem 3.6 constructed from $\mathscr{R}_{N}$. We define a finite state automaton $\mathscr{A}(C)$ by modifying $\mathscr{A}_{N}$ so that the only accepting states are those corresponding to the regions of $\mathscr{R}_{N}$ in $P$. By the comment after the proof of Proposition 3.4 an expression $w=s_{1} \cdots s_{k}$ is accepted by $\mathscr{A}(C)$ if and only if it is reduced and $w \in C$. This completes the proof.

As remarked before, each two-sided cell contains only finitely many left cells [31. Moreover, any right cell $C_{R}$ is obtained by inverting some left cell $C$, which means $\operatorname{Red}\left(C_{R}\right)$ consists of all words in $\operatorname{Red}(C)$ reversed. Since both a finite union of regular languages and the reversal of a regular language are regular [23, §4.2], we obtain the following corollary:

4.7. Corollary. Let $C$ be any cell in $\widetilde{W}$, including right and two-sided. Then $\operatorname{Red}(C)$ is regular. 
For practical computation, instead of $\operatorname{Red}(\widetilde{W})$ one usually works with a subset that attaches a unique reduced expression to every element of $\widetilde{W}$. Such a subset is provided by the shortlex expressions. Fix an ordering on the generators $\widetilde{S}$, and let $w=s_{1} \cdots s_{k}$ be a reduced expression. Then this expression is a shortlex expression for $w$ if for each $i$, the generator $s_{i}$ is the least element of $\widetilde{S}$ occurring in a reduced expression for $w$.

Brink and Howlett [11] showed that the language ShortLex $(\widetilde{W})$ of shortlex expressions for $\widetilde{W}$ is regular (as before, the results of [11] apply to all Coxeter groups, not just affine Weyl groups). We can prove a similar result for Kazhdan-Lusztig cells:

4.8. Corollary. Let $C$ be any cell in $\widetilde{W}$, including right and two-sided. Then ShortLex $(C)$ is regular.

Proof. By Corollary 4.7, the language $\operatorname{Red}(C)$ is regular. We have ShortLex $(C)=$ $\operatorname{Red}(C) \cap \operatorname{ShortLex}(\widetilde{W})$, and the result follows since the intersection of two regular languages is regular [23, $\S 4.2]$.

\section{REMARKS}

We conclude the paper by giving some comments on Theorem 4.6 and an example of its implementation.

5.1. It is interesting to consider the minimal value of $N$ needed to simultaneously show that all the cells of $\widetilde{W}$ give regular languages. A consequence of Shi's work 34 is that $N=0$ suffices for type $A$, and Figure 2 shows that $N=1$ is the minimal value needed for $\widetilde{G}_{2}$. Figure 2 also shows that the arrangement $\mathscr{H}_{1}$ contains extra lines not needed to decompose left cells into regions. For instance, the left- and rightmost vertical lines, as well as the highest and lowest horizontal lines, are not supporting boundary lines for any left cell, and thus are not needed to distinguish left cells from each other.

Thus one has the natural question of how to define the smallest possible automaton needed to describe all the left cells of $\widetilde{W}$. Such an automaton would have theoretical value, since using it one could attempt to extend Shi's work for type $A$ [34 to all types, especially the classical types (cf. 2, 13, 14, 15]). The obvious idea is to eliminate some hyperplanes from $\mathscr{H}_{N}$, such as the aforementioned lines in Figure 2. However, one must be careful to ensure that the resulting set of regions still satisfies property $(*)$. For instance, in Figure 2 one could try eliminating the outermost vertical lines, since they are clearly not needed to distinguish left cells. But then to keep property $(*)$ one will be forced to remove other lines from the arrangement, and some of these lines are necessary to separate left cells.

Hence, one must compromise: some extra hyperplanes can be deleted, but some must remain to preserve property $(*)$. Based on Remark 3.7 and examples, we raise the following question:

5.2. Question. Define $\nu: \Phi^{+} \rightarrow \mathbb{Z}_{\geq 0}$ by $\nu(\alpha)=0$ if $\alpha$ is short and $\nu(\alpha)=1$ if $\alpha$ is long. Let $\mathscr{H}_{\nu}$ be the affine hyperplane arrangement from (4), and let $\mathscr{R}_{\nu}$ be the connected components of the complement $V \backslash \mathscr{H}_{\nu}$. Then is any left cell of $\widetilde{W}$ a union of regions from $\mathscr{R}_{\nu}$ ? 


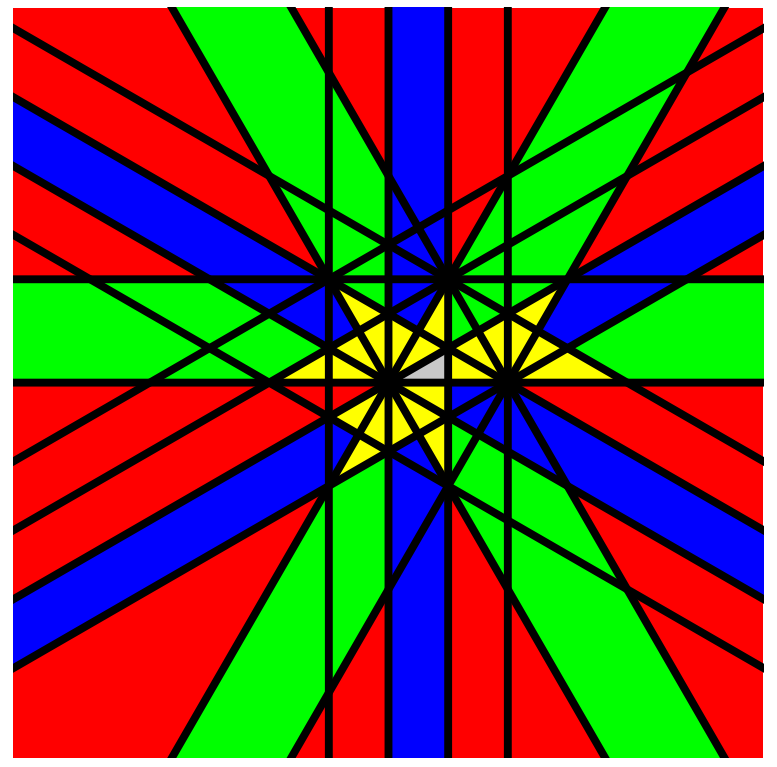

FiguRE 3 . The regions in $\mathscr{R}_{\nu}$ for $\widetilde{G}_{2}$

We have checked that the answer to Question 5.2 is yes for $\widetilde{C}_{2}$ (Figure 44), $\widetilde{G}_{2}$ (Figure 3), $\widetilde{C}_{3}$, and for the canonical left cells of $\widetilde{B}_{3}$. Note that an affirmative answer to this question for $\Phi$ simply-laced implies that sign-type regions are sufficient to describe the Kazhdan-Lusztig cells for $\Phi$.

5.3. Example. Here we give an example of an automaton for the root system $\widetilde{C}_{2}$ constructed using Proposition [3.4 and show how it can be used to recognize $\operatorname{Red}(C)$ for any cell $C$. To avoid extraneous states, we use the set of regions $\mathscr{R}_{\nu}$ from Question [5.2. These figures were produced using Perl [38, fsm [32], and graphviz 17.

The generating set $\widetilde{S} \subset \widetilde{W}$ contains three involutions $s_{0}, s_{1}, s_{2}$ satisfying the relations $\left(s_{0} s_{1}\right)^{4}=\left(s_{0} s_{2}\right)^{2}=\left(s_{1} s_{2}\right)^{4}=1$. Figure 4 shows the regions $\mathscr{R}_{\nu}$, labelled from 0 to 48. The gray region $R_{0}$ is the basic alcove $A_{0}$. The three purple lines along the edges of $A_{0}$ are the fixed point sets for the reflections in $\widetilde{S} ; s_{0}$ corresponds to the horizontal line, $s_{2}$ to the vertical line, and $s_{1}$ to the line with angle $\pi / 4$. The two-sided cells are colored red, green, yellow, and gray. To simplify the picture, we have not drawn the alcoves.

As in Proposition 3.4 the regions in $\mathscr{R}_{\nu}$ can be first used to build an automaton $\mathscr{A} \operatorname{recognizing} \operatorname{Red}(\widetilde{W})$. Figure 5 shows $\mathscr{A}$. There are 49 states in $\mathscr{A}$, labelled $q_{0}$ to $q_{48}$. State $q_{i}$ is connected to $q_{j}$ by an arrow labelled $s \in \widetilde{S}$ if the regions $R_{i}$ and $R_{0}$ lie on the same side of the $s$-purple line and if the reflection $s$ takes $R_{i}$ into $R_{j}$. (In Figure 5 we have omitted $q$ and $s$ from the vertices and arrows.) For instance, in the center of the figure there is an arrow $q_{27} \rightarrow q_{38}$ labelled $s_{1}$. The initial state of $\mathscr{A}$ is $q_{0}$, and all states of $\mathscr{A}$ are accepting if one wants to recognize $\operatorname{Red}(\widetilde{W})$. 
Now suppose one wants to recognize $\operatorname{Red}(C)$ for $C$ a left cell. For example let $C$ be the green left cell in the upper left of Figure 4 This cell is the union of the regions $R_{4}, R_{7}, R_{20}, R_{21}, R_{27}$. Thus we modify $\mathscr{A}$ by making the accepting states $q_{4}, q_{7}, q_{20}, q_{21}, q_{27}$. These states are indicated in Figure 5 by double circles. We build words in $\operatorname{Red}(C)$ by following paths that terminate in one of these states. The path

$$
q_{0} \longrightarrow q_{2} \longrightarrow q_{9} \longrightarrow q_{7} \longrightarrow q_{8} \longrightarrow q_{15} \longrightarrow q_{27},
$$

for instance, shows that $s_{1} s_{0} s_{2} s_{1} s_{0} s_{2}$ is an expression for an element of $C$.

For every regular language, there is a minimal automaton accepting that it is uniquely determined up to isomorphism. As the reader might guess, the automaton in Figure 5 is far from minimal. Figure 6 shows the minimal automaton accepting $\operatorname{Red}(C)$; as before $q_{0}$ is the initial state, and this time the only accepting state is $q_{4}$. From this figure it is not hard to see that $\operatorname{Red}(C)$ consists of the reduced expressions for the words $w$ that have a reduced expression containing either $s_{0} s_{2}$ or $s_{2} s_{0}$ as a subword, have right descent set $\left\{s_{0}, s_{2}\right\}$, and have no reduced expressions containing $s_{0} s_{1} s_{0}, s_{1} s_{0} s_{1}, s_{1} s_{2} s_{1} s_{2}$, or $s_{2} s_{1} s_{2} s_{1}$ as subwords. In other words, this is a subset of the reduced expressions for the fully commutative elements of $\widetilde{C}_{2}$ [37. This result is consistent with the conjectural description of the cells of Coxeter groups generated by reflections in the sides of a triangle given by the author with M. Belolipetsky [5]. We remark that Shi has investigated cells built of fully commutative elements and has proved that for $\widetilde{C}_{n}$, the fully commutative elements form a union of two-sided cells [36.

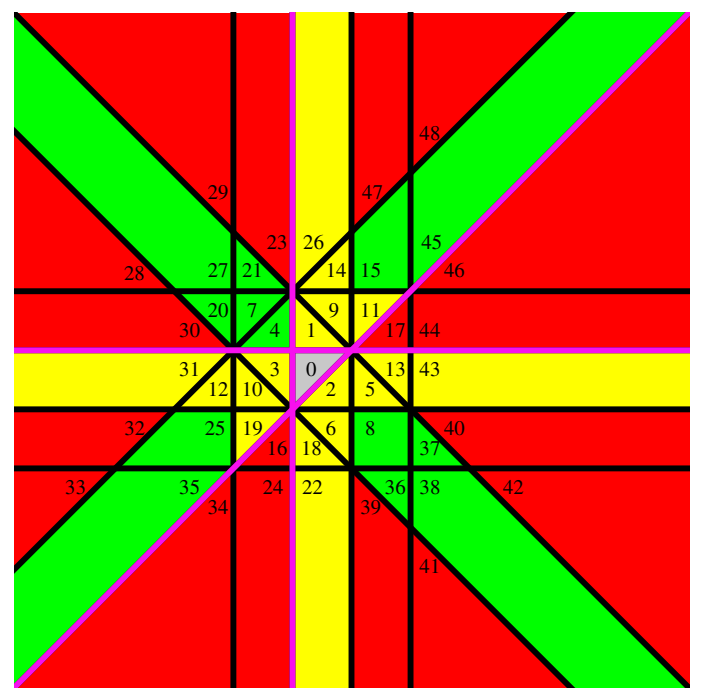

FiguRE 4. States for the automaton accepting $\operatorname{Red}\left(\widetilde{C}_{2}\right)$ built using $\mathscr{R}_{\nu}$ 


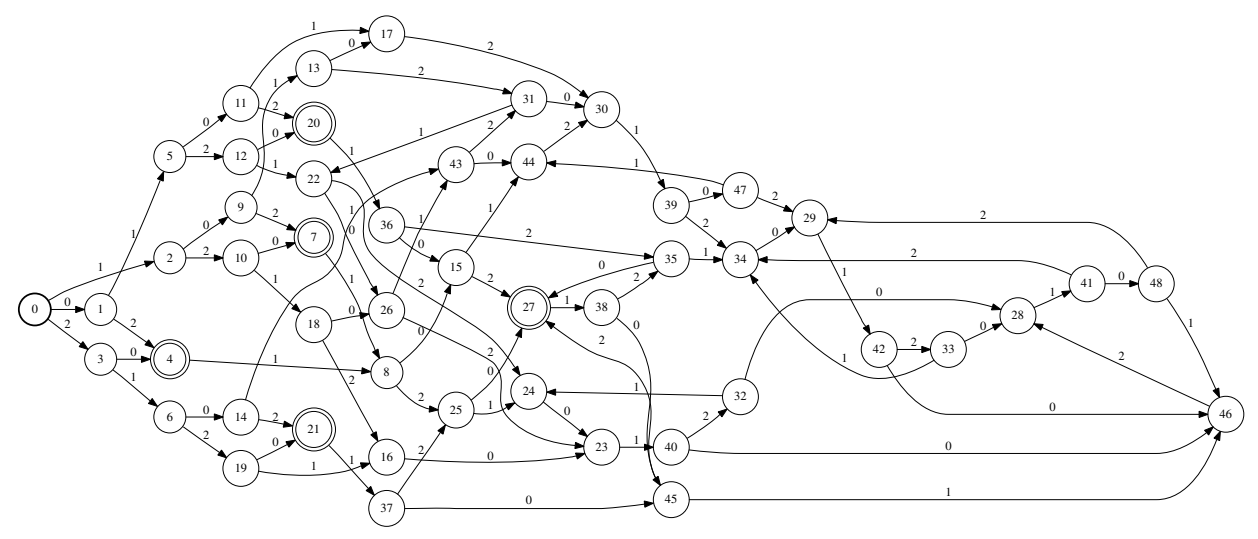

Figure 5. The automaton built from Figure 4

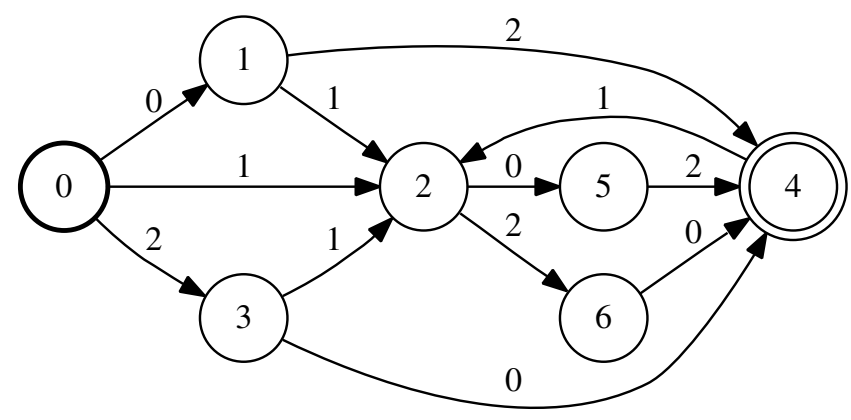

Figure 6 . The minimal automaton accepting $\operatorname{Red}(C)$

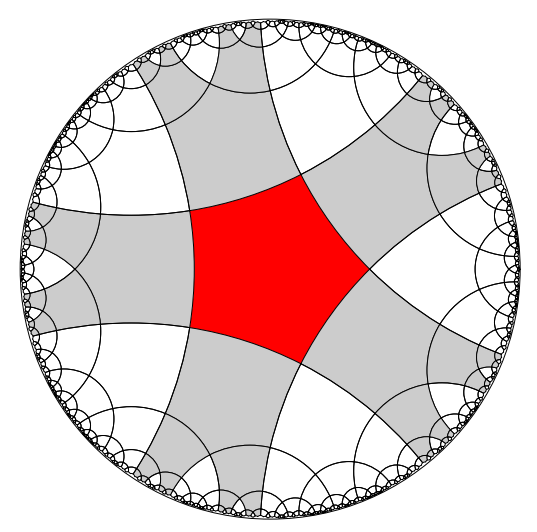

Figure 7. Cells for a hyperbolic Coxeter group 


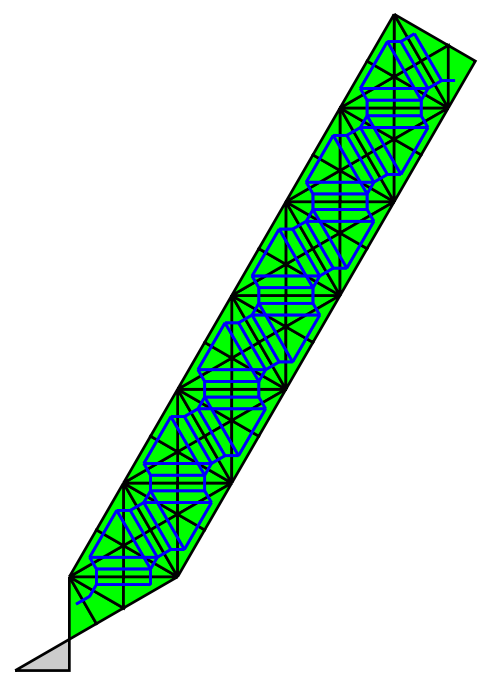

$$
\ell=1
$$

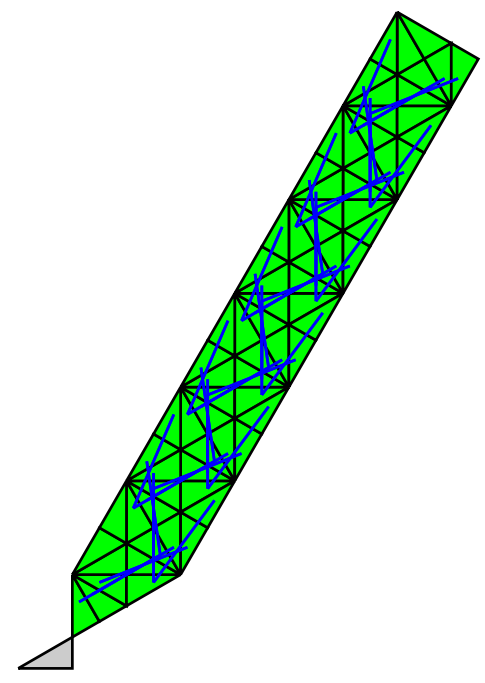

$$
\ell=5
$$

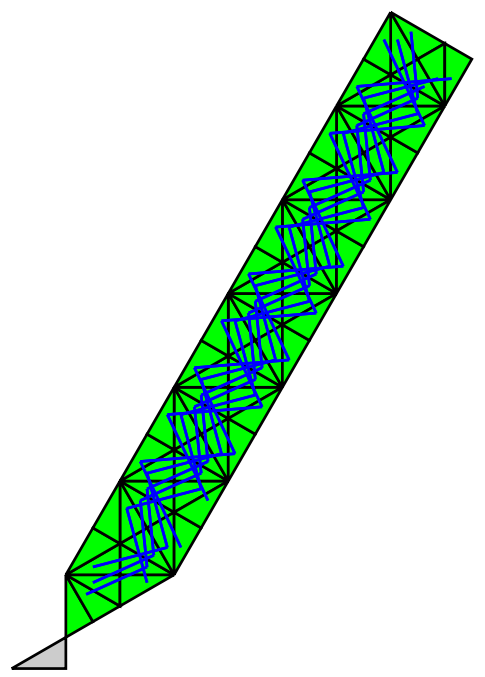

$$
\ell=3
$$

Figure 8

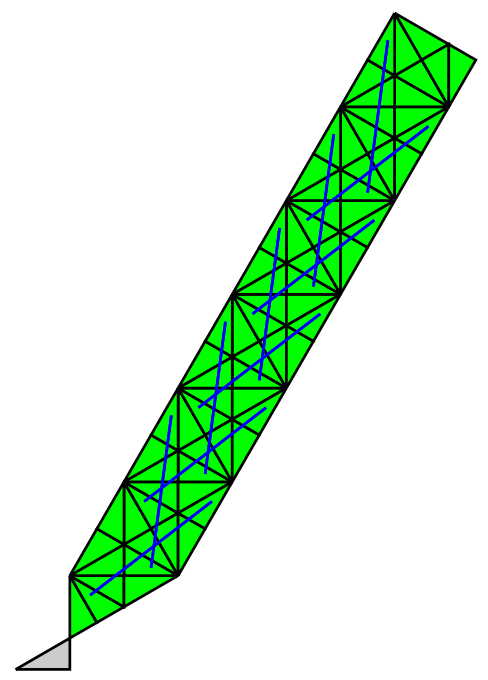

$$
\ell=7
$$

FiguRE 9 


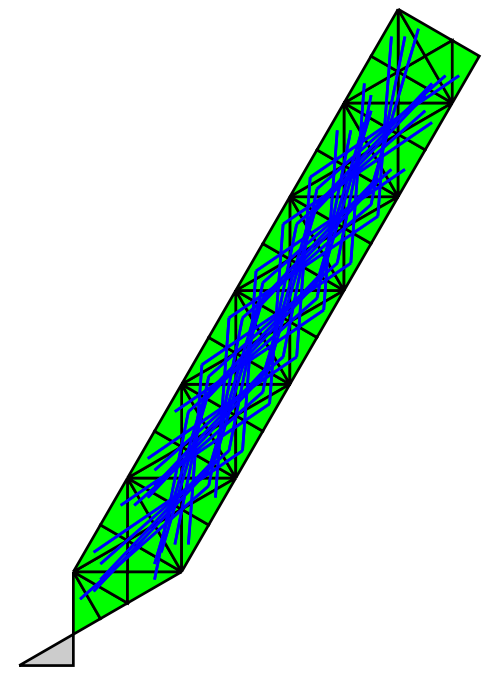

$\ell=9$

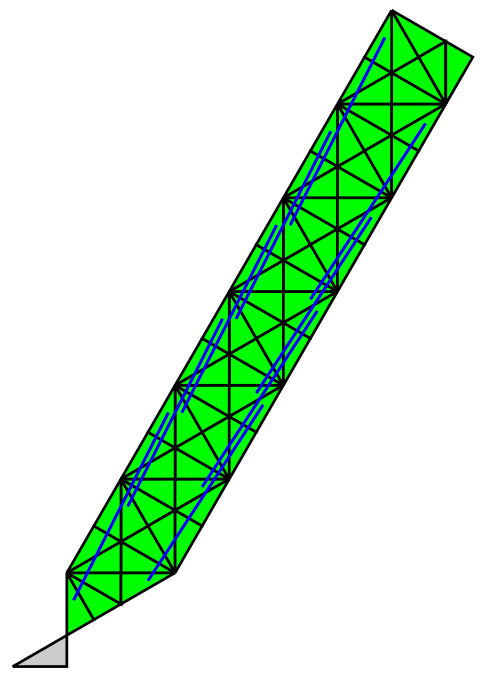

$\ell=11$

FigURE 10

5.4. Returning to the general case, one can ask if Theorem 4.6 and the stronger Question 5.2 apply to the case of cells with unequal parameters [27. For instance, J. Guilhot [19] has recently given a conjectural description of the left cells in $\widetilde{G}_{2}$ for all choices of parameters, and has proved them in many cases. For all the examples in his conjectural description, the answer to Question 5.2 is yes, and Theorem 4.6 also holds. Unfortunately, further computations of J. Guilhot 20] show that the answer to Question 5.2 is no for $\widetilde{C_{2}}$. Nevertheless, Theorem 4.3 and thus Theorem 4.6 both hold true in this case as well (in fact, one can take $N=1$ ).

5.5. Finally, it is natural to ask if the ideas in this paper can be used to prove Conjecture 1.3 for all Coxeter groups. For a general group, the analogue of the hyperplane arrangement $\mathscr{H}_{0}$ is the arrangement of minimal or small roots. This is a certain finite subset of the set of all root hyperplanes in the geometric realization of $W$ that plays the decisive role in proving the regularity of $\operatorname{Red}(W)$. Then one could try to identify a large finite subset of hyperplanes containing the minimal ones that could be used to determine an analogous class of polyhedra $\mathscr{P}$.

Unfortunately the naive generalization of the ideas in this paper cannot work. Belolipetsky [6] computed the cells of right-angled Coxeter groups generated by reflections in the hyperbolic plane about the sides of right-angled polygons. He showed that such groups have infinitely many left cells and three two-sided cells. The simplest example, which is typical, is the Coxeter group generated by reflections of a hyperbolic pentagon (Figure 7). The three two-sided cells are shown in red, gray, and white. It is clear that the white two-sided cell cannot be written as a finite union of convex polyhedra. Moreover, in Figure 7 the three gray left cells are bounded by infinitely many root hyperplanes, and thus cannot be written as the union of regions attached to a finite arrangement. 


\section{ACKNOWLEDGEMENTS}

We thank M. Belolipetsky, C. Bonnafé, W. Casselman, J. Guilhot, and J. Humphreys for helpful comments. We also thank M. Belolipetsky for Figure 7. We thank the referee for a very careful reading and for suggestions that improved the paper. The results in this paper were discovered through computation using modified versions of various programs due to W. Casselman, D. Holt, and F. du Cloux. This work was partially supported by the NSF through grants DMS 04-01525, 06-19492, 08-01214.

\section{REFERENCES}

[1] C. A. Athanasiadis, Algebraic combinatorics of graph spectra, subspace arrangements, and Tutte polynomials, Ph.D. thesis, MIT, 1996.

[2] R. Bédard, Cells for two Coxeter groups, Comm. Algebra 14 (1986), no. 7, 1253-1286. MR842039 (87h:20066)

[3] - The lowest two-sided cell for an affine Weyl group, Comm. Algebra 16 (1988), no. 6, 1113-1132. MR939034 (89d:20041)

[4] , Left V-cells for hyperbolic Coxeter groups, Comm. Algebra 17 (1989), no. 12, 29712997. MR1030605 (91a:20051)

[5] M. Belolipetsky and P. E. Gunnells, Experiments and conjectures for planar hyperbolic Coxeter groups, in preparation.

[6] M. Belolipetsky, Cells and representations of right-angled Coxeter groups, Selecta Math. (N.S.) 10 (2004), no. 3, 325-339. MR2099070 (2006f:20048)

[7] R. Bezrukavnikov, On tensor categories attached to cells in affine Weyl groups, Representation theory of algebraic groups and quantum groups, Adv. Stud. Pure Math., vol. 40, Math. Soc. Japan, Tokyo, 2004, pp. 69-90. MR2074589(2006e:20086)

[8] R. Bezrukavnikov and V. Ostrik, On tensor categories attached to cells in affine Weyl groups. II, Representation theory of algebraic groups and quantum groups, Adv. Stud. Pure Math., vol. 40, Math. Soc. Japan, Tokyo, 2004, pp. 101-119. MR2074591(2006e:20006)

[9] A. Björner and F. Brenti, Combinatorics of Coxeter groups, Graduate Texts in Mathematics, vol. 231, Springer, New York, 2005. MR2133266 (2006d:05001)

[10] N. Bourbaki, Éléments de mathématique. Fasc. XXXIV. Groupes et algèbres de Lie. Chapitre IV: Groupes de Coxeter et systèmes de Tits. Chapitre V: Groupes engendrés par des réflexions. Chapitre VI: systèmes de racines, Actualités Scientifiques et Industrielles, No. 1337, Hermann, Paris, 1968. MR0240238 (39:1590)

[11] B. Brink and R. B. Howlett, A finiteness property and an automatic structure for Coxeter groups, Math. Ann. 296 (1993), no. 1, 179-190. MR.1213378 (94d:20045)

[12] W. A. Casselman, Automata to perform basic calculations in Coxeter groups, Representations of groups (Banff, AB, 1994), CMS Conf. Proc., vol. 16, Amer. Math. Soc., Providence, RI, 1995, pp. 35-58. MR:1357194 (96i:20050)

[13] C. D. Chen, The decomposition into left cells of the affine Weyl group of type $\widetilde{D}_{4}$, J. Algebra 163 (1994), no. 3, 692-728. MR1265858 (95b:20063)

[14] J. Du, The decomposition into cells of the affine Weyl group of type $\widetilde{B}_{3}$, Comm. Algebra 16 (1988), no. 7, 1383-1409. MR941176 (89i:20074)

[15] Cells in the affine Weyl group of type $\widetilde{D}_{4}$, J. Algebra 128 (1990), no. 2, 384-404. MR.1036398 (91a:20052)

[16] - Sign types and Kazhdan-Lusztig cells, Chinese Ann. Math. Ser. B 12 (1991), no. 1, 33-39. MR.1098597 (92c:20089)

[17] J. Ellson, E. Gansner, E. Koutsofios, S. North, and G. Woodhull, Graphviz and dynagraph static and dynamic graph drawing tools, Graph Drawing Software (M. Junger and P. Mutzel, eds.), Springer-Verlag, 2003, pp. 127-148.

[18] H. Eriksson, Computational and combinatorial aspects of Coxeter groups, Ph.D. thesis, KTH Stockholm, 1994.

[19] J. Guilhot, Kazhdan-Lusztig cells in affine Weyl groups with unequal parameters, Ph.D. thesis, Université Claude Bernard Lyon I and Aberdeen University, 2008.

[20] , personal communication, 2009. 
[21] P. E. Gunnells, Cells in Coxeter groups, Notices Amer. Math. Soc. 53 (2006), no. 5, 528-535. MR2254399 (2007d:20068)

[22] P. Headley, Reduced expressions in infinite Coxeter groups, Ph.D. thesis, University of Michigan, 1994

[23] J. E. Hopcroft and J. D. Ullman, Introduction to automata theory, languages, and computation, Addison-Wesley Publishing Co., Reading, Mass., 1979, Addison-Wesley Series in Computer Science. MR645539 (83j:68002)

[24] J. E. Humphreys, Reflection groups and Coxeter groups, Cambridge Studies in Advanced Mathematics, vol. 29, Cambridge University Press, Cambridge, 1990. MR 1066460 (92h:20002)

[25] D. Kazhdan and G. Lusztig, Representations of Coxeter groups and Hecke algebras, Invent. Math. 53 (1979), no. 2, 165-184. MR560412 (81j:20066)

[26] _ Schubert varieties and Poincaré duality, Geometry of the Laplace operator (Proc. Sympos. Pure Math., Univ. Hawaii, Honolulu, Hawaii, 1979), Proc. Sympos. Pure Math., XXXVI, Amer. Math. Soc., Providence, R.I., 1980, pp. 185-203. MR573434 (84g:14054)

[27] G. Lusztig, Left cells in Weyl groups, Lie group representations, I (College Park, Md., 1982/1983), Lecture Notes in Math., vol. 1024, Springer, Berlin, 1983, pp. 99-111. MR727851 (85f:20035)

[28] - Some examples of square-integrable representations of p-adic semisimple groups, Trans. Amer. Math. Soc. 277 (1983), 623-653. MR694380 (84j:22023)

[29] — Characters of finite groups of Lie type, Ann. of Math. Studies, vol. 107, Princeton University Press, 1984. MR742472 (86j:20038)

[30] Cells in affine Weyl groups, Algebraic groups and related topics (Kyoto/Nagoya, 1983), Adv. Stud. Pure Math., vol. 6, North-Holland, Amsterdam, 1985, pp. 255-287. MR 803338 (87h:20074)

[31] Cells in affine Weyl groups. II, J. Algebra 109 (1987), no. 2, 536-548. MR902967 $(88 \mathrm{~m}: 20103 \mathrm{a})$

[32] M. Mohri, F. C. N. Pereira, and M. D. Riley, ATEST Finite State Machine Library, available from AT\&T Labs.

[33] A. Postnikov and R. P. Stanley, Deformations of Coxeter hyperplane arrangements, J. Combin. Theory Ser. A 91 (2000), no. 1-2, 544-597, In memory of Gian-Carlo Rota. MR 1780038 (2002g:52032)

[34] J. Y. Shi, The Kazhdan-Lusztig cells in certain affine Weyl groups, Lecture Notes in Mathematics, vol. 1179, Springer-Verlag, Berlin, 1986. MR835214(87i:20074)

[35] _ Sign types corresponding to an affine Weyl group, J. London Math. Soc. (2) 35 (1987), no. 1, 56-74. MR871765 (88g:20103b)

[36] J.-Y. Shi, Fully commutative elements and Kazhdan-Lusztig cells in the finite and affine Coxeter groups, Proc. Amer. Math. Soc. 131 (2003), no. 11, 3371-3378 (electronic). MR.1990625 (2004d:20044)

[37] J. R. Stembridge, On the fully commutative elements of Coxeter groups, J. Algebraic Combin. 5 (1996), no. 4, 353-385. MR1406459 (97g:20046)

[38] L. Wall, The Perl Programming Language, v5.8.8.

Department of Mathematics and Statistics, University of Massachusetts, Amherst, MASSACHUSETTS 01003

E-mail address: gunnells@math.umass.edu 